\title{
Breast implant-associated anaplastic large cell lymphoma (BIA-ALCL) - How to diagnose and treat?
}

\author{
Karolina Pieszko ${ }^{1}$, Maciej Kuczyński², Dawid Murawa³
}

Breast implant-associated anaplastic large cell lymphoma ( $A L C L)$ is a rare type of T-cell non-Hodgkin lymphoma arising around the capsule of breast implants. It has been diagnosed in an extremely small group of women with breast implants for breast reconstruction and augmentation. The pathogenesis of this disease is currently poorly understood, but it appears to be related to textured implants. The aim of this article is to provide patients, radiologists, pathologists, surgical oncologists and plastic surgeons with an evidence-based overview of the incidence, diagnosis, and management of BIA-ALCL according to real-world experience, because although it is very rare, early recognition and surgical resection is usually crucial and curative.

NOWOTWORY J Oncol 2018; 68, 1: 15-21

Key words: breast implants, lymphoma, large-cell, anaplastic, incidence, seroma, prognosis, algorithms, consent forms

\section{Introduction}

Breast implant-associated anaplastic large cell lymphoma (BIA-ALCL) is an uncommon entity arising primary in the capsule of breast prostheses. The disease represents only $2-3 \%$ of non-Hodgkin lymphomas and $12 \%$ of T-cell lymphomas. Tumors are characterized by the presence of a monoclonal expansion of CD30+ large anaplastic or Reed-Sternberg cells and are Anaplastic Lymphoma Kinase (ALK) negative [1].

Since 1962, silicone gel prostheses have been in use for esthetic operations and according to Bizjak et al. over 10 million augmentations with implants have been performed [2]. The first case of BI-ALCL was only reported in 1997 [3].

In this article, we collected and analyzed data on BIA-ALCL, such as pathophysiology, risk factors, presentation, diagnosis, treatment, outcomes and prevention. The PubMed and Medline databases were searched using the following keywords: breast implants, lymphoma, large-cell, anaplastic, incidence, seroma, prognosis, algorithms, consent forms. All review articles, case reports, original research articles, and any other articles relevant to BIA-ALCL were included.

\section{Etiology and prevalence}

The association between breast implants and anaplastic large cell lymphoma was indicated by the Food and Drug Administration in the United States in 2011. The latest findings reveal that the organization is aware of 359 adverse events reports of BIA-ALCL. 232 of these have included information on the breast implant material (203 implants identified as textured, 28 as smooth, and one as "another surface"). Both silicone gel and saline implants have been reported in cases of BIA-ALCL [4]. Since then, other case reports and series have been published with the largest report of 173 patients from around the world published in 2015 by Brody et al. [5]. The rate of diagnosis is rising with time, which is probably because of the influence of increased use of implants, and increasing clinician awareness and education.

It is estimated, that 1-3 cases per 1,000,000 women with implants per year develop BI-ALCL and although the

\footnotetext{
${ }^{1}$ Department of Plastic Surgery and Burns, Hospital in Nowa Sól, Poland

${ }^{2}$ Dr Maciej Kuczyński's Plastic Surgery Centre, Lublin, Poland

${ }^{3}$ Department of General and Minimally Invasive Surgery, Baptism of Poland Memorial Hospital, Gniezno, Poland
} 
rate is rare, it has been documented worldwide $[6,7]$. However, recent reports from the Australian Therapeutic Goods Administration state that the incidence of BIA-ALCL is significantly higher, between 1 case per 1000 and 1 case per 10,000 women who have breast implants in Australia [8]. The true incidence is unknown; it is undoubtedly increasing. According to de Jong et al., the odds ratio for ALCL of breast developing in women exposed to breast implants is 18.2 times greater than in those not carrying breast implants of any size [9]. In systematic review by Leberfinger et al., the mean age at onset of BIA-ALCL was 51 years, most women had breast implants in place for a mean of approximately 10 years before diagnosis (8.6 years for reconstruction vs 9.9 years for cosmetic surgery) [10]. The incidence of BIA-ALCL may be underestimated. It is important to determine risk factors and carry out more epidemiological studies which could be reported in a single official registry. Instead of defining a clear etiology of this disease, several risk factors are suggested and based on them some hypotheses have been created.

The most common factors like the presence of a subclinical biofilm on the implant surface, capsular contracture, repeated capsular trauma, genetic predisposition, or an autoimmune etiology have been theorized [9]. Another article has also hypothesized toxic damage from the silicone component [11]. The immune system's response to chronic inflammation surrounding the breast implant in a genetically susceptible patient may lead to genetic degeneration and dysplasia [1].

Nowadays, the proportion of used textured implants breast augmentation and reconstruction exceeds the number of smooth shell devices but this can not explain exhaustively why almost all cases of BI-ALCL have arisen in association with textured implants. The texture of implant surface varies by manufacturer, and can generally be classified as macrotextured, including polyurethane and Biocell, intermediate-textured or microtextured. Higher bacterial counts and greater T-cell response have been demonstrated in association with the more aggressive macrotextured implants [9].

The implant biofilm can promote chronic T-cell activation surrounding breast implants in genetically predisposed women [12], which may be the inciting factor in BIA-ALCL development. Tissue ingrowth into the pores of textured implants is thought to prolong chronic inflammation and CD4 T-cells have been found to be the predominant cell type [13]. Additionally, it was demonstrated by Kadin et al. that patterns of cytokine and transcription factor expression are suggestive of a Th1 phenotype, further supporting the theory that BIA-ALCL may arise from chronic bacterial antigen stimulation of T cells [14]. That is why it is expected that bacterial contamination and biofilm formation is minimized during surgery. In the case of capsular contracture, this has been achieved through the "14-point effect" $[15,16]$. The Surgical 14-Point Plan for Breast Implant Placement proposed by Adams et al. is presented in detail in Table I [17].

According to the data of reducing the number of bacteria around implants using this technique, it has been checked if aside from minimizing the occurrence of capsular contracture to less than $1 \%$ [18], the technique can also influence the incidence of BIA-ALCL.

A prospective study has been carried out by eight plastic surgeons in five countries by placing about 43,000 macrotextured breast implants using similar techniques. Surgeons adhered to at least 13 steps from the "14-Point Plan for Breast Implant Placement". Respectively, mean follow-up

Table I. The proposed Surgical 14-Point Plan for Breast Implant Placement [17]

1. Use intravenous antibiotic prophylaxis at the time of anesthetic induction

2. Avoid periareolar/transaxillary incisions; these have been shown in both laboratory and clinical studies to lead to a higher rate of contracture

3. Use nipple shields to prevent spillage of bacteria into the pocket

4. Perform careful atraumatic dissection to minimize devascularized tissue

5. Perform careful prospective hemostasis

6. Avoid dissection into the breast parenchyma

7. Use a dual-plane pocket

8. Perform pocket irrigation with correct proven betadine triple-antibiotic solution, non-betadine triple or $50 \%$ ( $1: 1$ dilution) or stronger povidone-iodine

9. Steps to minimize skin contamination (e.g. wipe/prep skin, barrier, sleeve)

10. Minimize implant open time and replacement of implant or sizers

11. Change surgical gloves before handling and use new or cleaned instruments and drapes

12. Avoid using a drainage tube, which can be a potential site of entry for bacteria (augmentation)

13. Use a layered closure

14. Use antibiotic prophylaxis to cover subsequent procedures that breach skin or mucosa 
was 11.7 (range, 1 to 14 years) in patients with Biocell and 8.0 years (range, 1 to 20 years) in patients with polyurethane implants. For primary breast augmentation, $77.9 \%$ of all implants were used, followed by augmentation mastopexy $9.9 \%$, revision augmentation $8.2 \%$, and breast reconstruction $4 \%$. The capsular contracture rate was $2.2 \%$. The expected number of the incidence of breast implant-associated $\mathrm{ALCL}$ in this study using only macrotextured breast implants would be between eight and nine diagnoses. However, there were no cases at all [17]. One may logically suspect that the operative technique is an important factor in the use of breast implants, especially those with the highest risk.

It has been proved that a pro-inflammatory environment can cause cancer. An example can be the association of Helicobacter pylori infection and gastric lymphoma. Recently, Hu et al., have reported an increased prevalence of bacteria called Ralstonia pickettii (a Gram-negative common contaminant of drinking water) within the microbiome of breast implant capsules taken from patients with BIA-ALCL compared to those with normal capsular contracture [19].

Loch-Wilkinson et al. identified a total of 55 patients from Australia and New Zealand between 2007 and 2016 showed the frequency of implant types associated with BI-ALCL. Forty-four women had a single-implant exposure and the remaining eleven had a multiple-implant exposure. That is why a total of 75 breast implant pairs were applied in this retrospective study. The mean age of the patients was 47.1 years (range, 22.4 to 69.6 years). The mean time to develop BIA-ALCL from the time of the last implantation was 7.46 years (range, 0.2 to 27.0 years). All patients were exposed to textured implants at some point in their implant history. Comparative analysis showed the risk of developing breast implant-associated ALCL to be higher with Biocell textured implants followed by polyurethane (Silimed) textured implants. Table II presents the frequency of BIA-ALCL in relation to implant type according to Loch-Wilkinson et al. [20].

Table II. Frequency of implant types associated with breast implantassociated ALCL [14]

\begin{tabular}{lll}
\hline Texture Type & Manufacturer & No. (\%) \\
\hline Biocell (salt loss) & Allergan/Inamed/McGhan & $44(58.7)$ \\
Polyurethane & Silimed & $14(18.7)$ \\
Salt loss & Nagor & $5(6.7)$ \\
Polyurethane & Surgitek & $1(1.3)$ \\
Siltex & Mentor & $5(6.7)$ \\
PIP & PIP & $2(2.7)$ \\
Smooth & Mentor & $2(2.7)$ \\
Smooth & Unknown & $2(2.7)$ \\
\hline
\end{tabular}

Despite published reports and study cases, according to the conclusions of the 2016 Aesthetic Breast Meeting in Milan about $\mathrm{BI}-\mathrm{ALCL}$, actual evidence does not permit establishment of any statistically significant association between implant, patient, surgery-related risk factors which could be drawn until large epidemiologic studies are conducted [21]. Therefore, future research is expected to determine whether certain patients and procedures have a predisposition to development of the disease.

\section{Presentation and staging of the disease}

BIA-ALCL patients typically present with malignant effusions associated with the fibrous capsule surrounding an implant (80\%) or with solid capsule-related masses (20\%), which may indicate a more aggressive clinical course [22].

These effusions are called in situ disease and are not a palpable breast tumor; they can be misinterpreted as a benign seroma due to subclinical infection. The median time after implantation to diagnose $A L C L$ is 9 years (range from 1 to 32 years). A lower number of patients suffer from an infiltrative disease course, with a palpable mass around the capsule with or without periprosthetic effusion. This can be associated with lymph node and bilateral breast involvement, which usually means a worse prognosis, with disease-related mortality as high as $40 \%$ in 2 years [23]. The mean size of well documented breast mass associated with $\mathrm{BI}-\mathrm{ALCL}$ is $3.5 \mathrm{~cm}$ [24].

Usually BI-ALCL cases are diagnosed during implant revision surgery which is performed for a late onset (more than 1 year). The majority of BI-ALCL cases have an indolent course provided adequate surgical ablation of the implant and surrounding capsule without systemic therapy, but there have also been reported aggressive exceptions, disease progression, and death [25], the disease may be associated with local symptoms such as pain (21\%), redness (14\%), capsular contracture (7\%), skin lesions (7\%), and fever (7\%) [7]. Symptoms of breast lumps, swelling or breast asymmetry may be also associated [25].

Clinical and pathological characteristics did not differ significantly between implant-exposed patients and breast-ALCL patients without implant exposure, except for seromaassociated features uniquely in patients with implants [26].

Patients with $\mathrm{BI}-\mathrm{ALCL}$ or any type of lymphoma are traditionally staged by the 1971 Ann Arbor Classification presented in Table III [27]. Under this classification, nearly all $\mathrm{BI}-\mathrm{ALCL}$ patients fall under one of two stages. The majority of patients (84\%) were Ann Arbor stage IE or II, the remaining patients (16\%) considered as stage IV with bone or muscle lesions [28]. In the other systematic review reported by Gidengil et al. in 2015, the most common stage at diagnosis is IE (61\%), followed by stage IIE (11\%). For the rest of patients, staging information in this study remains unknown [29]. 
Table III. Ann Arbor staging system

\begin{tabular}{ll}
\hline Staging & Description \\
\hline Stage I & $\begin{array}{l}\text { Involvement of a single lymph node region or single lymphoid structure, such as spleen, thymus or Waldeyer ring (I), or a single } \\
\text { extranodal site (IE) }\end{array}$ \\
Stage II & $\begin{array}{l}\text { Involvement of two or more lymph node regions or lymphoid structures on the same side of the diaphragm (II) or localized } \\
\text { involvement of an extralymphatic site and one or more lymph nose regions on the same side of the diaphragm (IIE) }\end{array}$ \\
Stage III & $\begin{array}{l}\text { Involvement of lymph nodes regions or lymphoid structures on both sides of the diaphragm (III), which can also be accompanied } \\
\text { by localized involvement of an extralymphatic site (IIIE), or spleen (IIIS) or both (IIISE) }\end{array}$ \\
Stage IV & $\begin{array}{l}\text { Diffuse or disseminated involvement of one or more extralymphatic organs or tissues with or without associated lymph node } \\
\text { enlargement }\end{array}$ \\
\hline
\end{tabular}

Breast implant-associated anaplastic large cell lymphoma behaves more like a solid tumor than a lymphoma. Therefore, Clemens et al. proposed using TNM staging, typically used for solid tumors, instead of the Ann Arbor staging classification.

The specific surgical and pathologic BIA-ALCL staging system, modeled after the American Joint Committee on Cancer TNM system is presented in Table IV. It divides patients into a very low risk group confined to the effusion or a layer on the luminal side of the capsule (stage I) and a higher risk group with an extra-luminal spread, a breast mass or distant metastases which need more aggressive systemic treatment. Patients with stage II are locally advanced and with stage III/IV disease appear regional and distant spread [7].

This staging differs from the commonly used Ann Arbor staging and appears to predict overall survival more accurately than the Ann Arbor system. Moreover, BI-ALCL seems to behave more similarly to other breast malignancies than to lymphomas with regard to the treatment, including surgical excision, and the clinical course of the disease [7].

\section{Diagnosis}

Diagnosis is made by the finding of abnormal cells in the aspirate or biopsy accompanied by the hallmark uniform over-expression of CD30 and negative staining for Anaplastic Lymphoma Kinase (ALK).

Clemens et al. [30] have described some specific criteria for diagnosis of breast implant-associated anaplastic large cell lymphoma such as:

1. Adequate specimen of a tumor, involving an effusion surrounding a breast implant

2. Large lymphoid cells with abundant cytoplasm and pleomorphic nuclei

3. T-cell markers with uniform expression of CD30 by immunohistochemistry or flow cytometry

4. Negative for anaplastic lymphoma kinase (ALK) protein or translocations involving the $A L K$ gene at chromosome $2 q 23$

For suspected patients, any aspiration of periprosthetic fluid should be sent to pathology for cytologic evaluation through fine-needle aspiration, flow cytometry, and CD30

Table IV. The proposed TNM Staging for Breast Implant-Associated Anaplastic Large-Cell Lymphoma [20]

\begin{tabular}{|c|c|c|c|}
\hline TNM or Stage Designation & & Description & \\
\hline \multirow[t]{4}{*}{ T: tumor extent } & $\mathrm{T} 1$ & Confined to effusion or a layer on luminal side of capsule & \\
\hline & $\mathrm{T} 2$ & Early capsule infiltration & \\
\hline & $\mathrm{T} 3$ & Cell aggregates or sheets infiltrating the capsule & \\
\hline & $\mathrm{T} 4$ & Lymphoma infiltrates beyond the capsule & \\
\hline \multirow[t]{3}{*}{ N: lymph node } & No & No lymph node involvement & \\
\hline & $\mathrm{N} 1$ & One regional lymph node (+) & \\
\hline & $\mathrm{N} 2$ & Multiple regional lymph nodes (+) & \\
\hline \multirow[t]{2}{*}{ M: metastasis } & M0 & No distant spread & \\
\hline & M1 & Spread to other organs/distant sites & \\
\hline \multirow[t]{7}{*}{ Stage } & IA & T1N0M0 & \multirow{3}{*}{ Low risk group } \\
\hline & $\mathrm{IB}$ & T2NOMO & \\
\hline & IC & T3N0M0 & \\
\hline & $\| A$ & T4NOMO & \multirow{4}{*}{ High risk group } \\
\hline & $\| \mathrm{B}$ & T1-3N1M0 & \\
\hline & III & T4N1-2M0 & \\
\hline & IV & TanyNanyM1 & \\
\hline
\end{tabular}


immunohistochemistry of effusion including a clinical history with the stated intent to "rule out BI-ALCL". Pathologic evaluation may demonstrate BI-ALCL as individual cells, cell clusters in aggregates, or as cohesive sheets. Diagnosis using Wright-Giemsa or hematoxylin and eosin staining alone is usually insufficient, however BI-ALCL will demonstrate strong and uniform membranous expression of CD30 immunohistochemistry [13].

Ultrasound may help define the extent of an effusion and can be helpful in identifying any associated capsule masses. Included in clinical examination should be evaluation of regional lymph nodes. The volume of an effusion can range from 50 to $1,000 \mathrm{~mL}$ and is typically more viscous than a benign seroma owing to the high protein content and cellularity. The surrounding capsule may be thickened and fibrous or may be deceptively unremarkable on gross examination consistent with the under-appreciation of this lymphoma. If a mass is present, it can protrude outward into the soft tissue or into the implant creating a mass effect distortion on imaging [5].

Adrada et al. reviewed 44 breast implant-associated $\mathrm{ALCL}$ cases according to radiologic imaging features and made a comparison according to image sensitivity and specificity of specific methods presented in Table V. Thus, it is recommendable to use ultrasound as a screening tool and for some cases also MRI or PET to determine extension and for surveillance of disease. In regard to mammography, its sensitivity was reported as insufficient for BI-ALCL effusion and mass [31].

\section{Management and treatment}

Mostly patients have slow disease progression and a good prognosis but it is important to pay attention in the case of occasional lymphadenopathy and metastases, which need also some adjuvant medical therapies.

According to two recommended management algorithms for BIA-ALCL in the USA and the UK $[6,30]$, doctors should diagnose and examine those patients presenting symptoms like sudden, unexplained effusion, new painful mass around or involving an implant capsule, especially at least one year after implant insertion.

Usually it is advisable to use ultrasounds for breast and ipsilateral lymph nodes. Moreover, it is mentioned in the
English algorithm to use mammogram, if there is residual breast tissue and in the American algorithm to use MRI if ultrasound is inconclusive.

In a case of effusion, it is recommendable to proceed with fine needle aspiration cytology (FNAC) of total effusion volume. This is different to presentation of a mass, which needs needle core biopsy (NCB) of the mass and or without abnormal axillary lymph nodes, followed by oncologic consultation. The next step is to diagnose all types of specimens using cytology, histology, flow cytometry and checking CD30 cells. Upon confirming histologically BIA-ALCL, anaplastic lymphoma kinase (ALK) negative, CD30 positive cells, it is crucial to report BIA-ALCL to the PROFILE Registry.

After reporting it is advisable to discuss the case within a multidisciplinary team consisting of a plastic surgeon, a surgical oncologist, an oncologist and a pathologist.

Lymphoma staging is very important for choosing the best treatment for the patient; this can be determined by PET, CT scan or bone marrow biopsy.

Patients with stage I (localized disease) are treated with implant removal and total capsulectomy. Those presenting stage II, III, IV (advanced disease), besides surgical treatment with mass and nodes excision with total capsulectomy and explantation, also need some adjuvant therapies, like chemotherapy or immunotherapy as decided by the multidisciplinary team $[6,30]$.

Standard chemotherapy regimens used in the management of systemic ALCL are anthracycline-based, such as cyclophosphamide, doxorubicin, vincristine and prednisolone (CHOP). This treatment seems to be more effective in patients with ALK-positive systemic ALCL when compared to ALK-negative disease (5-year survival rates of 70 to $90 \%$ vs 40 to $60 \%$ ). In patients with ALK negative systemic disease, $\mathrm{CHOP}$ may be ineffective. Other regimens may be used and remissions consolidated with autologous stem cell transplantation [25].

Clinical trials assessing the use of Brentuximab Vedotin, is a novel anti-CD30 monoclonal antibody that has improved the management of ALK-negative systemic ALCL with a reported objective response rate of $86 \%$ and complete remission rate of $59 \%$ in relapsed or refractory systemic ALCL [32].

Table V. Comparison of imaging sensitivity and specificity of specific methods [23]

\begin{tabular}{lcccc}
\hline $\begin{array}{l}\text { RADIOLOGIC IMAGING } \\
\text { FEATURES }\end{array}$ & EFFUSION & & BI-ALCL MASS \\
\hline Ultrasound & sensitivity & specificity & sensitivity & $100 \%$ \\
Computerized Tomography & $84 \%$ & $75 \%$ & $46 \%$ & $50 \%$ \\
Magnetic Resonance Imaging & $55 \%$ & $83 \%$ & $33 \%$ & $82 \%$ \\
Positron Emission Tomography & $82 \%$ & $83 \%$ & $64 \%$ & $88 \%$ \\
\hline
\end{tabular}


Recently, Fleming et al. described two interesting cases of documented evidence of spontaneous regression and spontaneous resolution of confirmed BIA-ALCL. The first patient, due to enlargement of the breast (after breast implant augmentation in 1994 and 2009), underwent aspiration, the cytology of which was diagnostic for BIA-ALCL with abundant atypical T cells that were CD30+ and ALKAfter two months, the aspiration was repeated and most of the cells identified were benign macrophages and flow cytometry was normal. The second case is a patient who underwent an uncomplicated primary breast augmentation in submuscular pockets in May 2013, and in February 2017 complained of a sudden enlargement of her breast. At the beginning, cytology and immunohistochemistry confirmed BIA-ALCL with atypical T cells which were CD30+ and ALK- . Next, she had a bilateral explantation and capsulectomies in May 2017, but cytology, flow cytometry and immunohistochemistry of a small residual fluid collection, and histopathology of the capsule, showed no evidence of malignancy. This not only proves that the disease can spontaneously resolve but also that it can do so rapidly. Nevertheless, given the potentially fatal consequences of inadequate treatment, and the present inability to be certain that the disease is not invasive without histopathology, bilateral explantation and capsulectomy should remain the current recommended minimum treatment [33].

\section{Conclusions}

The aim of this article was to raise awareness through education and better information about BIA-ALCL. It is crucial to remember that each seroma occurring more than 1 year after implantation not confirmed by infection or trauma should be considered as suspicious for disease. However, we should remember that many benign causes for the sudden development of peri-implant fluid exist and these are the majority compared to BIA-ALCL. It is recommended that cases are managed in specialist tertiary centers which have the appropriate experience in the management of the disease. In the BIA-ALCL context, although the risk is extremely low, it is nevertheless doctors' duty to provide information to all patients considering breast implant surgery and include BIA-ALCL among possible complications in the informed consent form. The American Society of Plastic Surgeons prepared an example of such a document, which is available for download from their website: www.plasticsurgery.org. Moreover, according to the recommendation of the consensus established during the Maurizio Bruno Nava (MBN) 2016 Aesthetic Breast Meeting, all confirmed BIA-ALCL cases should be reported to the Patient and Outcomes for Breast Implants and Anaplastic Large Cell Lymphoma Etiology and Epidemiology and to the respective competent authorities that regulate or guarantee the safety of medical devices [21]. Undoubtedly, current knowledge about BIA-ALCL patho- genesis, diagnostic pathways, prognosis, and therapeutic options is limited. Therefore, reporting and more analytical epidemiologic studies will provide better evidence on the disease in the future.

\section{Conflict of interest: none declared}

\section{Karolina Pieszko, MD}

Hospital in Nowa Sól Poland

Department of Plastic Surgery and Burns

Chałubińskiego $7 \mathrm{St}$.

67-100 Nowa Sól, Poland

e-mail:karolina@pieszko.pl

Received: 26 Jan 2018

Accepted: 10 Mar 2018

We thank Dr Elżbieta Parka-Barańska and Dr Andrzej Barański for invaluable inspiration with this study.

\section{References}

1. Kaartinen I, Sunela K, Alanko J et al. Breast implant-associated anaplastic large cell lymphoma - from diagnosis to treatment. Eur J Surg Oncol 2017; 43: 1385-1392.

2. Bizjak M, Selmi C, Praprotnik S et al. Silicone implants and lymphoma: The role of inflammation. J Autoimmun 2015; 65: 64-73.

3. Keech JA, Creech BJ. Anaplastic T-cell lymphoma in proximity to a saline-filled breast implant. Plast Reconstr Surg 1997; 100: 554-555.

4. The Center for Devices and Radiological Health USFaDA Anaplastic large cell lymphoma (ALCL) in women with breast implants: Preliminary FDA findings and analysis. http://www.fda.ogv/downloads/medicaldevices/ productsandmedicalprocedures/implantsnadprosthetics/breastimplants/UCM240003.pdf.

5. Brody GS, Deapen D, Taylor CR et al. Anaplastic large cell lymphoma occurring in women with breast implants: analysis of 173 cases. Plast Reconstr Surg 2015; 135: 695-705.

6. Johnson L, O'Donoghue JM, McLean N et al. Breast implant associated anaplastic large cell lymphoma: the UK experience. Recommendations on its management and implications for informed consent. Eur J Surg Oncol 2017; 43: 1393-1401.

7. Clemens MW, Medeiros LJ, Butler CE et al. Complete surgical excision is essential for the management of patients with breast implant-associated anaplastic large-cell lymphoma. J Clin Oncol 2016; 34: 160-168.

8. Breast implants and anaplastic large cell lymphoma. Australian Goverment Department of Health Website [cited 2018 Mar 7]. Available from: https: //www.tga.gov.au/alert/breast-implants-and-anaplasticlarge-cell-lymphoma.

9. de Jong D, Vasmel WL, de Boer JP et al. Anaplastic large-cell lymphoma in women with breast implants. JAMA 2008; 300: 2030-2035.

10. Leberfinger AN, Behar BJ, Williams NC et al. Breast implant-associated anaplastic large cell lymphoma: A systematic review. JAMA Surg 2017; 152: 1161-1168.

11. Ye X, Shokrollahi K, Rozen WM et al. Anaplastic large cell lymphoma (ALCL) and breast implants: breaking down the evidence. Mutat Res Rev Mutat Res 2014; 762: 123-132.

12. Ferreri AJ, Govi S, Pileri SA et al. Anaplastic large cell lymphoma, ALK-positive. Crit Rev Oncol Hematol 2012; 83: 293-302.

13. Hart AM, Lechowicz MJ, Peters KK et al. Breast implant-associated anaplastic large cell lymphoma: Report of 2 cases and review of the literature. Aesthetic Surg J 2014; 34: 884-894.

14. Kadin ME, Deva A, Xu H et al. Biomarkers provide clues to early events in the pathogenesis of breast implant-associated anaplastic large cell lymphoma. Aesthetic Surg J 2016; 36: 773-781.

15. Deva AK, Adams WP, Vickery K. The role of bacterial biofilms in device-associated infection. Plast Reconstr Surg 2013; 132: 1319-1328.

16. Adams WP. Capsular contracture: what is it? What causes it? How can it be prevented and managed? Clin Plast Surg 2009; 36: 119-126.

17. Adams WP, Culbertson EJ, Deva AK et al. Macrotextured breast implants with defined steps to minimize bacterial contamination around the device: experience in 42,000 implants. Plast Reconstr Surg 2017; 140: 427-431. 
18. Adams WP, Rios JL, Smith SJ. Enhancing patient outcomes in aesthetic and reconstructive breast surgery using triple antibiotic breast irrigation: Six-year prospective clinical study. Plast Reconstr Surg 2006; 118 (7 Suppl): 46S-52S.

19. Hu H, Jacombs A, Vickery $K$ et al. Chronic biofilm infection in breast implants is associated with an increased T-cell lymphocytic infiltrate: implications for breast-implant-associated lymphoma. Plast Reconstr Surg 2015; 135: 319-329.

20. Loch-Wilkinson A, Beath KJ, Knight RJW et al. Breast implant-associated anaplastic large cell lymphoma in Australia and New Zealand: high-surface-area textured implants are associated with increased risk. Plast Reconstr Surg 2017; 140: 645-654.

21. Nava MB, Adams WP, Botti G et al. MBN 2016 Aesthetic Breast Meeting BIA-ALCL Consensus Conference Report. Plast Reconstr Surg 2018, 141: 40-48.

22. Olack B, Gupta R, Brooks GS. Anaplastic large cell lymphoma arising in a saline breast implant capsule after tissue expander breast reconstruction. Ann Plast Surg 2007; 59: 56-57.

23. Laurent C, Delas A, Gaulard P et al. Breast implant-associated anaplastic large cell lymphoma: two distinct clinicopathological variants with different outcomes. Ann Oncol 2016; 27: 306-314.

24. Kim B, Roth C, Chung KC et al. Anaplastic large cell lymphoma and breast implants: a systematic review. Plast Reconstr Surg 2011;127: 2141-2150.

25. Kim B, Roth C, Young VL et al. Anaplastic large cell lymphoma and breast implants: results from a structured expert consultation process. Plast Reconstr Surg 2011; 128: 629-639.
26. de Boer $\mathrm{M}$, van Leeuwen $\mathrm{FE}$, Hauptmann $\mathrm{M}$ et al. Breast implants and the risk of anaplastic large-cell lymphoma in the breast. JAMA Oncol 2018; 4: 335-341.

27. Carbone PP, Kaplan HS, Musshoff $\mathrm{K}$ et al. Report of the Committee on Hodgkin's Disease Staging Classification. Cancer Res 1971; 31: 1860-1861.

28. Laurent C, Delas A, Gaulard P et al. Breast implant-associated anaplastic large cell lymphoma: two distinct clinicopathological variants with different outcomes. Ann Oncol 2016; 27: 306-314.

29. Gidengil CA, Predmore Z, Mattke $S$ et al. Breast implant-associated anaplastic large cell lymphoma: a systematic review. Plast Reconstr Surg 2015; 135: 713-720.

30. Clemens MW, Nava MB, Rocco N et al. Understanding rare adverse sequelae of breast implants: anaplastic large-cell lymphoma, late seromas, and double capsules. Gland Surg 2017; 6: 169-184.

31. Adrada BE, Miranda RN, Rauch GM et al. Breast implant-associated anaplastic large cell lymphoma: sensitivity, specificity, and findings of imaging studies in 44 patients. Breast Cancer Res Treat 2014; 147: 1-14.

32. Younes A, Bartlett NL, Leonard JP et al. Brentuximab vedotin (SGN35) for relapsed CD30-positive lymphomas. N Engl J Med 2010; 363: 1812-1821.

33. Fleming D, Stone J, Tansley P. Spontaneous regression and resolution of breast implant-associated anaplastic large cell lymphoma: implication for research, diagnosis and clinical management. Aesthetic Plast Surg 2018 [Epub ahead of print]. 